\title{
Editorials
}

\section{Approaches to diagnosing cancer earlier in general practice}

In the UK there have been significant efforts over the last two decades to reduce delays in cancer diagnosis in response to the nation's poorer survival outcomes compared to other high-income countries. Cancer screening programmes are one approach to improve survival through early detection, but the majority of cancers are detected following symptomatic presentation in primary care. Theoretical models of diagnostic delay emphasise the importance of considering patient, health system, and tumour factors along a pathway from symptom appraisal and help seeking to diagnostic assessment and treatment. ${ }^{\prime}$ Such models identify potential points of intervention to improve the timeliness of cancer diagnosis.

\section{SYMPTOM AWARENESS INTERVENTIONS}

Improving community symptom awareness and reducing barriers to early help seeking is one such approach. Several symptom awareness campaigns have occurred in the UK with some evidence, from before-and-after data, of positive effects on presentations to health care, cancer diagnoses, and earlier stage. ${ }^{2}$ In contrast, a controlled trial of a community symptom awareness intervention in rural Australia failed to demonstrate reduced time to presentation in people subsequently diagnosed with cancer. ${ }^{3}$ There remains some doubt about the effectiveness and cost-effectiveness of community awareness campaigns; they are relatively expensive and may not reach those most at risk of poorer cancer outcomes.

In this issue, Laake et al report a more targeted approach in patients at risk of later diagnosis based on socioeconomic status and past consulting behaviours. ${ }^{4}$ They trialled a letter from their general practice that highlighted six 'red-flag' cancer symptoms and promoted help seeking. This relatively simple, low-cost intervention

"Achieving timely diagnosis of cancer will continue to be an important priority for general practice. This is especially the case currently given the consequences of the COVID-19 pandemic on diagnostic and treatment delays.

led to more general practice consultations and higher rates of investigations. These findings are consistent with the trial results of a behavioural intervention to promote GP consultation in patients at increased risk of lung cancer. ${ }^{5}$ Although neither trial was large enough to demonstrate effects on cancer diagnoses, they suggest that targeted symptom awareness interventions can increase help seeking, even in populations that are considered hard to reach. They could therefore contribute to an overall strategy to improve the timeliness of cancer diagnosis.

\section{GUT FEELINGS AND CLINICAL INTUITION}

From a general practice perspective, it is well recognised that certain cancers are more challenging to diagnose and are associated with multiple GP consultations before referral. ${ }^{6}$ These are cancers that have non-specific symptoms and are less likely to fit cancer referral criteria. A second article in this issue discusses the concept of a GP's gut feelings and how they contribute to cancer diagnosis in general practice, especially for these more challenging types of clinical presentation.? Friedemann Smith et al describe the "grey area' between normal and abnormal, and how GPs interpret symptoms in the context of their knowledge of the individual patient and their clinical experience. Gut feelings,

\section{"Theoretical models of diagnostic delay emphasise the importance of considering patient, health system, and tumour factors along a pathway from symptom appraisal and help seeking to diagnostic assessment and treatment.}

perhaps also considered as clinical intuition, can result in a patient falling in the grey area where the GP assesses symptoms as serious even though they may not reflect cancer referral criteria. Clinical guidelines and referral pathways need to be adapted to account for these gut feelings and recognise their potential to select patients requiring prompt investigation.

Gut feelings may be particularly relevant when considering the diagnosis of multiple myeloma. This relatively uncommon cancer is often associated with delays in diagnosis and multiple GP consultations. ${ }^{6}$ Patients with myeloma have non-specific symptoms that will commonly have been investigated with routine blood tests, but the diagnosis not considered and serum or urine electrophoresis therefore not ordered.

\section{RISK PREDICTION MODELS}

A third article in this issue reports the development and validation of a risk prediction model for myeloma. ${ }^{8}$ Koshiaris et al used data from the Clinical Practice Research Datalink to develop a risk model that included symptoms (back, rib or chest pain, and nosebleeds), full blood count parameters (haemoglobin, white cell count, platelets, and mean cell volume), age, and sex. They showed that this model was more sensitive and specific in identifying patients with myeloma than symptoms or single test results such as anaemia. The low prevalence of myeloma means that, even using this risk prediction model, a GP would have to test 271 patients to diagnose one case, but this highlights the need for a low threshold for investigation for cancers with common nonspecific symptoms. The authors discuss the potential to implement the myeloma risk model into a decision support tool within the GP electronic medical record. This could help prompt GPs to consider the diagnosis and investigate sooner. 


\section{“A combination of approaches aimed at reducing barriers to patient presentation, application of risk prediction models, and respect for a GP's clinical intuition should all play a part in our goal to improve cancer outcomes.}

There is some evidence that computer decision support tools can improve cancer diagnosis in general practice but there remain significant challenges to their implementation into routine care, including integration with GP software and ensuring they fit within GP workflow. 9.10

\section{THE FUTURE OF EARLY DETECTION OF CANCER}

Achieving timely diagnosis of cancer will continue to be an important priority for general practice. This is especially the case currently given the consequences of the COVID-19 pandemic on diagnostic and treatment delays. ${ }^{11}$ Cancer Research UK has recently published a roadmap to the future of early detection of cancer. ${ }^{12}$ The report emphasises the need to improve risk stratification in cancer screening programmes and the development of novel artificial intelligence-based algorithms within the electronic medical record for earlier diagnosis of cancer in symptomatic patients. It also highlights the growing role of genomics and other novel biomarker tests to underpin risk assessment of both asymptomatic and symptomatic populations. The articles reported in this edition of the BJGP all contribute to this broader vision of how to improve early diagnosis of cancer in general practice. A combination of approaches aimed at reducing barriers to patient presentation, application of risk prediction models, and respect for a GP's clinical intuition should all play a part in our goal to improve cancer outcomes.

\section{Jon D Emery}

Herman Professor of Primary Care Cancer Research, Department of General Practice and Centre for Cancer Research, University of Melbourne, Australia.

\section{Provenance}

Commissioned; externally peer reviewed.

\section{Competing interests}

The authors have declared no competing interests.

DOI: https://doi.org/10.3399/bjgp21X715613

\section{ADDRESS FOR CORRESPONDENCE}

\section{Jon D Emery}

Centre for Cancer Research, University of Melbourne, 10th floor, Victorian Comprehensive Cancer Centre, 305 Grattan St, Parkville, Vic 3010, Australia.

\section{Email: jon.emery@unimelb.edu.au}

\section{REFERENCES}

1. Scott SE, Walter FM, Webster A, et al. The model of pathways to treatment: conceptualization and integration with existing theory. Br J Health Psychol 2013; 18(1): 45-65.

2. Kennedy MPT, Cheyne L, Darby M, et al. Lung cancer stage-shift following a symptom awareness campaign. Thorax 2018; 73(12): 1128-1136.

3. Emery JD, Gray V, Walter FM, et al. The Improving Rural Cancer Outcomes Trial: a cluster-randomised controlled trial of a complex intervention to reduce time to diagnosis in rural cancer patients in Western Australia. $\mathrm{Br} \mathrm{J}$ Cancer 2017; 117(10): 1459-1469.

4. Laake J-P, Vulkan D, Quaife S, et al. Targeted encouragement of GP consultations for possible cancer symptoms: randomised controlled trial. Br J Gen Pract 2021; DOI: https://doi. org/10.3399/bjgp20X713489.

5. Emery JD, Murray SR, Walter FM, et al. The Chest Australia Trial: a randomised controlled trial of an intervention to increase consultation rates in smokers at risk of lung cancer. Thorax 2019; 74(4): 362-370.

6. Lyratzopoulos G, Neal RD, Barbiere JM, et al. Variation in number of general practitioner consultations before hospital referral for cancer: findings from the 2010 National Cancer Patient Experience Survey in England. Lancet Oncol 2012; 13(4): 353-365

7. Friedemann Smith C, Moller Kristensen B, Sand Andersen $\mathrm{R}$, et al. GP' use of gut feelings when assessing cancer risk. Br J Gen Pract 2021; DOI: https://doi.org/10.3399/bjgp21X714269.

8. Koshiaris C, Van den Bruel A, Nicholson BD, et al. Clinical prediction tools to identify patients at highest risk of myeloma in primary care: a retrospective open cohort study. Br J Gen Pract 2021; DOI: https://doi.org/10.3399/ BJGP.2020.0697.

9. Chima S, Reece JC, Milley K, et al. Decision support tools to improve cancer diagnostic decision making in primary care: a systematic review. Br J Gen Pract 2019; DOI: https://doi. org/10.3399/bjgp19X706745

10. Bradley PT, Hall N, Maniatopoulos G, et al. Factors shaping the implementation and use of Clinical Cancer Decision Tools by GPs in primary care: a qualitative framework synthesis. BMJ Open 2021; 11(2): e043338.

11. Lai $A G$, Pasea L, Banerjee A, et al. Estimated impact of the COVID-19 pandemic on cancer services and excess 1-year mortality in people with cancer and multimorbidity: near real-time data on cancer care, cancer deaths and a population-based cohort study. BMJ Open 2020; 10(11): e043828.

12. Cancer Research UK. Early detection and diagnosis of cancer. A roadmap to the future. London: Cancer Research UK. 2021. 